\title{
The Clinical Effects of Epidural Anethesia with Lidocaine Combined Low Dose Ropivacaine in Elective Cesarean Section
}

\author{
Huijun Wang ${ }^{1}$, Yin $\mathrm{Bao}^{1}$ and Jun Xiong ${ }^{2 *}$ \\ ${ }^{1}$ Department of Anesthesiology, Capital Medical University Beijing Tongren Hospital. No.1 Dongjiao Minxiang, China
}

${ }^{2}$ Department of Anesthesiology, Sanbo Brain Hospital, Capital Medical University. No. 50 Yikesong, China

*Corresponding author: Jun Xiong, Department of Anesthesiology, Sanbo Brain Hospital, Capital Medical University. No. 50

Yikesong, Xiangshan, Haidian District, Beijing, 100093, China

ARTICLE INFO
Received: 蔧 May 01, 2021
Published: 冓 June 15, 2021

Citation: Huijun Wang, Yin Bao, Jun Xiong. The Clinical Effects of Epidural Anethesia with Lidocaine Combined Low Dose Ropivacaine in Elective Cesarean Section. Biomed J Sci \& Tech Res 36(3)-2021. BJSTR. MS.ID.005866.

Keywords: Lidocaine; Ropivacaine; Epidural Anesthesia; Spinal Anesthesia; Cesarean Section

Abbreviations: CS: Cesarean Section; CSEA: Combined Spinal-Epidural Anesthesia; DBP: Diastolic Blood Pressure; ECS: Elective Cesarean Section; EA: Epidural Anesthesia; HR: Heart Rate; SA: Spinal Anesthesia; SBP: Systolic Blood Pressure

\section{ABSTRACT}

Objective: To observe the effects of epidural anesthesia with lidocaine combined low dose ropivacaine in elective cesarean section, and to provide more effective and simple anesthetic methods for obstetrics.

Methods: 60 women undergoing elective cesarean section were randomly divided into two groups, combined spinal-epidural anesthesia group (CSEA group, $n=27$ ), and lidocaine and ropivacaine group (LR group, $\mathrm{n}=33$ ). Combined spinal-epidural anesthesia with hyperbarci ropivacaine was used in CSEA group, and epidural anesthesia with lidocaine combined low dose ropivacaine was used in LR group. Monitoring and recording basic SBP, DBP and HR of all cases (T0). The lowest SBP, DBP and the fastest HR were recorded (T1), and the SBP, DBP and HR of 30min after anesthesia (T2) were recorded too. The anesthetic effects were evaluated by Anesthetic Effect Score and modified Bromage score. Meanwhile adverse reactions were recorded.

Results: There was no difference on SBP, DBP and HR of T0 and T2 of two groups $(\mathrm{P}>0.05)$. Compared to CSEA group, SBP and DBP of T1 of LR group were increased significantly $(\mathrm{P}<0.05)$, and HR of $\mathrm{T} 1$ in two group was similar $(\mathrm{P}>0.05)$. The lowest SBP in both groups were on T1, the highest SBP of two groups were on T0, following T2. DBP and HR in two groups on T0 and T2 were similar. But DBP on T1 was the lowest in two group, and HR on T1 was the highest in two groups. There was no difference on Anesthetic Effect Score in two groups. Compared to CSEA group, modified Bromage score of LR group was reduced significantly at the end of anesthesia and the end of operation $(\mathrm{P}<0.05)$. Compared to CSEA group, the duration of anesthesia manuevre was reduced significantly, meanwhile, the onset of operation was reduced significantly $(\mathrm{P}<0.05)$. The cases of nausea were significantly reduced in LR groups compared to CSEA group $(\mathrm{P}<0.05)$. The scores of Apgar of two groups in $1 \mathrm{~min}$ and $5 \mathrm{~min}$ were indifferent $(\mathrm{P}>0.05)$.

Conclusion: The clinical effect of epidural anesthesia with lidocaine combined low dose ropivacaine was reliable. With this anesthesia, circulation of maternity was more stable, adverse reaction and the extents of movement blocked were reduced too. So it was more effective and simple anesthesia method for cesarean section.

\section{Background}

Ropivacaine is a long-acting amide type local anesthetic which has a greater degree of separation between motor and sensory blockade. Because of its less central nerves system and cardiovascular adverse toxicity, it has been most generally used in obstetrical anesthesia and postoperative pain relief in our hospital. Another reason for its being suitable in obstetrical anesthesia is 
little impact on uterus, placenta and maternal hemodynamics [1] Spinal anesthesia (SA) is preferred anesthetic method for cesarean section (CS) not only in China [2], but also in other worldwide regions in fact. However there are some of disadvantages of SA including nausea, hemodynamic disorders such as hypotension [3]. Hypotension induced by SA is still a common complication [4], which might be up to $80 \%$ [5]. Such high incidence is the main limitation of SA in CS [6]. Although ropivacaine has more stable hemodynamics effect, it might induce hypotension in SA, especially with high doses in obese parturient women [7]. Thus small dose of local anesthestic was advocated due to low incidence of hypotension induced by SA, however it possibly increased the failures [8,9]. In some cases phenylephrine has to be administered because of its' more advantages to prevent SA related to hypotension $[5,10]$.

Epidural anesthesia (EA) is another anesthesia management choice for CS. However from 1991 anesthesia service for elective cesarean section (ECS) changed from EA to SA due to advocated better and more cost effective SA [11]. Recently a retrospective comparative study indicated that analgesia agent was more required in SA than EA, and there was no difference related to length of hospital stay and operative time between them. Even in emergency, EA would be recommended [12]. Additionally, lidocaine has rapid onset of anesthesia than ropivacaine and is much cheaper [13], in some extent lidocaine is able to make up for the delayed onset of EA.

This randomized study was to compare the clinical effects between two anesthesia approaches in patients undergoing ECS, EA with lidocaine combining low dose ropivacaine and combined spinal-epidural anesthesia (CSEA) with ropivacaine, and provide more suitable anesthesia method for CS.

\section{Methods}

\section{Patients Recruitment}

This prospective, randomized, single-blinded study included sixty patients scheduled for ECS were recruited from March of 2017 to October of 2018.The inclusion criteria was pregnancy over 38 weeks and less than 42 weeks, ASA class I-II and scheduled ECS. The exclusion criteria were as follow: hypertension, multiple pregnancies, once SA or EA, presence of SA or EA contraindications, ASA class higher than II, all indications of emergency CS and abnormalities of embryo.

\section{Study Protocols}

Using random number generator, all parturient patients were randomly enrolled into two groups: $2.5 \mathrm{ml}$ intrathecal hyperbaric 0.5\% ropivacaine (AstraZeneca Co., Ltd., 2022-04NBDS.) (CSEA group) and EA with $18 \mathrm{ml} 2 \%$ lidocaine (Double-Crane Co., Ltd., H11022295.) combined $4 \mathrm{ml}$ 0.375\% ropivacaine (LR group) All patients were monitored and recorded continuously with electrocardiogram, noninvasive blood pressure and pulse oximetry. Oxygen was routinely given by mask at $3 \mathrm{~L} / \mathrm{min}$ rate. After building forearm venous access with 18 gauge cannula, all parturient women were infused with $5 \mathrm{ml} / \mathrm{Kg}$ Lactated Ringer's solution within 10min. CSEA and EA were administrated in left lateral decubitus position under aseptic precautions. Epidural puncture was performed in L3 and L4 intervertebral space with Durasafe Adjustable BD needle. Intradermal injection of $2 \%$ lidocaine was administrated, punctured skin and superficial tissue by a 20 gauge introducer needle. Midline approach with the standard technique of loss of resistance to saline was implemented. $3 \mathrm{ml} 2 \%$ lidocaine was injected into epidural space, if without syndromes of SA, $15 \mathrm{ml}$ $2 \%$ lidocaine was injected into epidural space over 30 s, followed by insertion epidural catheter into epidural space $4 \mathrm{~cm}$.

CSEA was performed with needle-through-needle technique getting to subarachnoid space. As cerebrospinal fluid free flow, $12.5 \mathrm{mg}$ ropivacaine was administered via spinal needle over 20s. After withdraw of the needle, epidural catheter was inserted into epidural space. All parturient patients were recovered to position of 15-degree tilt to left side after anesthesia maneuver. In LR group, $4 \mathrm{ml}$ 0.375\% ropivacaine was administered through epidural catheter as soon as position recovery. If sensory blockade level was not achieved at T6 on the beginning of operation, $2 \%$ lidocaine was administered via epidural catheter in $3 \mathrm{ml}$ increment with $5 \mathrm{~min}$ intervals to reach the level. If systolic blood pressure (SBP) decreased by $20 \%$ of baseline or below $90 \mathrm{mmHg}, 50 \mathrm{ug}$ phenylephrine was administered. If heart rate was monitored less than $60 \mathrm{bpm}, 0.5 \mathrm{mg}$ atropine was given intravenously. If blockade level on $\mathrm{T} 6$, but the patient required additional anesthetic, or experienced visceral traction response and stomach discomfort, 5ug sulfentanyl was administered intravenously. Nausea of patients was treated with granisetron. All ECS were performed by the same obstetricians, and the same anesthesiologist performed all anesthesia procedures, but all patients were monitored and evaluated by another anesthesiologist.

\section{Assessment and Data Collection}

Hemodynamic parameters included SBP and diastolic blood pressure (DBP), these and heart rate (HR) were recorded before onset of anesthesia as the baseline values (T0). After completion of anesthesia, the lowest SBP and DBP, and the highest HR were recorded as T1. Thirty minutes after the completion of anesthesia, SBP, DBP and HR were T2. When the patient was on left lateral decubitus position preparing to accept anesthesia, the time was recorded as the beginning of anesthesia. The duration of anesthesia, the onset and duration of operation were recorded respectively. The motor and sensory blockade levels evaluation were performed at $2 \mathrm{~min}$ intervals for the first $30 \mathrm{~min}$ and recorded up to the end of operation. Motor block was detected with modified Bromage score, $0=$ no paralysis, $1=$ unable to raise extended leg, $2=$ unable 
to flex knee and 3=unable to flex ankle. The sensory changes were assessed bilaterally along midclavicular line by pinprick test. The effect of anesthesia included analgesia, visceral traction response and abdominal muscle relaxation, which was classified into three levels [14]. Agpar scores were used to assess all neonates' physical condition on $1 \mathrm{~min}$ and $5 \mathrm{~min}$ after fetal delivery. Additionally, side effects, such as nausea and vomiting, were recorded, as well intraoperative supplemental doses and times of lidocaine and sulfentanyl.

\section{Statistical Analysis}

Statistical analysis was performed with IBM SPSS for Windows 21.0 (IBM, Beijing, China). Numerical variables were expressed with mean and standard deviation $\bar{x} \pm s$. Categorical data were presented with numbers. Normal distribution was verified with Shapiro-Wilk method. Normally distributed means were compared with independent-samples t test or one-way analysis of variance (ANOVA), in this procedure homogeneity of variance was tested firstly. Categorical data was analyzed with $\chi^{2}$ test. If the expected number in any cell of contingency table was less than five, Fisher's exact test was used. $\mathrm{P}<0.05$ was considered as statistical significant.

\section{Results}

Parturient women characteristics were shown on Table 1.

On T0 and T2, SBP, DBP and HR of both groups were similar $(\mathrm{P}>0.05)$. On T1, SBP and DBP of LR group were significantly higher than these of CSEA group $(\mathrm{P}<0.05)$, but the difference of HR in two groups was not significant. In both groups, the highest SBP was on T0, followed by $\mathrm{T} 2$, and SBP of T1 was the lowest $(\mathrm{P}>0.05)$. DBP and $\mathrm{HR}$ in both groups were similar on $\mathrm{T} 0$ and $\mathrm{T} 2$, but DBP on these time was higher than that of $\mathrm{T} 1(\mathrm{P}<0.05)$, and HR on these time was lower than that of $\mathrm{T} 1(\mathrm{P}<0.05)$ (Table 2). The Anesthetic Effect scores of LR group were as same as those of CSEA group ( $\mathrm{P}>0.05)$. However, comparing with CSEA group, the modified Bromage scores of LR group were significantly lower on the end of anesthesia and on the end of operation $(\mathrm{P}<0.05)$ (Table 3$)$. At the end of operation, the cases of nausea in LR group were significantly lower than CSEA group $(\mathrm{P}<0.05)$. Although operation duration of both groups were similar, the duration of local anesthesia and onset of operation in LR group were significantly shorter comparing to CSEA group $(\mathrm{P}<0.05)$ (Table 4). And there was no significant difference in Apgar score of neonates in two groups $(\mathrm{P}>0.05)$ (Table 5).

Table 1: The profile of all parturient patients.

\begin{tabular}{|c|c|c|c|c|c|}
\hline & N & Age (Y) & Height (cm) & Weight (Kg) & Gestational age (W) \\
\hline CSEA & 27 & $32.63 \pm 4.60$ & $165.26 \pm 4.05$ & $78.81 \pm 7.26$ & $39.48 \pm 1.12$ \\
\hline LR & 33 & $32.94 \pm 3.98$ & $164.85 \pm 3.28$ & $79.97 \pm 7.24$ & $39.18 \pm 0.88$ \\
\hline$t$ & & -0.279 & 0.434 & -0.614 & 1.158 \\
\hline$P$ & & 0.781 & 0.666 & 0.542 & 0.251 \\
\hline
\end{tabular}

Table 2: SBP, DBP and HR on T0, T1 and T2 in two groups.

\begin{tabular}{|c|c|c|c|c|c|c|}
\hline \multirow{2}{*}{} & \multicolumn{2}{|c|}{ SBP $(\mathbf{m m H g})$} & \multicolumn{2}{c|}{ DBP $(\mathbf{m m H g})$} & \multicolumn{2}{c|}{ HR $(\mathbf{b p m})$} \\
\cline { 2 - 7 } & CSEA & LR & CSEA & LR & CSEA & LR \\
\hline T0 & $131.33 \pm 6.96$ & $132.48 \pm 6.09$ & $72.15 \pm 8.79$ & $72.79 \pm 7.05$ & $84.07 \pm 8.63$ & $83.33 \pm 8.32$ \\
\hline T1 & $99.07 \pm 4.76^{\# \&}$ & $107.58 \pm 6.39^{* \# \&}$ & $57.30 \pm 4.91^{\# \&}$ & $64.03 \pm 8.33^{* \# \&}$ & $100.41 \pm 132.40^{\# \&}$ & $95.39 \pm 10019^{\# \&}$ \\
\hline T2 & $117.44 \pm 6.07^{\#}$ & $115.45 \pm 5.45^{\#}$ & $70.15 \pm 6.07$ & $69.58 \pm 6.76$ & $82.78 \pm 3.98$ & $84.88 \pm 4.59$ \\
\hline
\end{tabular}

Note: *Compared to CSEA group, SBP and DBP of LR group were significantly higher on T1 (P<0.05); \#compared to T0, SBP and DBP of T1 in both groups were significantly lower, SBP and DBP of T2 in both groups were significantly lower too, HR of T1 in both groups were significantly higher, $(\mathrm{P}<0.05)$; \&compared to T2, SBP and DBP of T1 in both groups were significantly lower, HR of TI in both groups were significantly higher $(\mathrm{P}<0.05)$.

Table 3: Anesthetic Effect scorle and Modified Bromage score of two group.

\begin{tabular}{|c|c|c|c|c|c|c|c|c|c|c|c|c|}
\hline & \multirow{3}{*}{$\mathbf{N}$} & \multirow{2}{*}{\multicolumn{3}{|c|}{ Anesthetic Effect scale }} & \multicolumn{8}{|c|}{ Modified Bromage Scale } \\
\hline & & & & & \multicolumn{4}{|c|}{ Anesthesia finishing } & \multicolumn{4}{|c|}{ End of opeation } \\
\hline & & 1 & 2 & 3 & 0 & 1 & 2 & 3 & 0 & 1 & 2 & 3 \\
\hline CSEA & 27 & 0 & 1 & 26 & 0 & 27 & 0 & 0 & 0 & 18 & 9 & 0 \\
\hline \multirow[t]{3}{*}{ LR } & 33 & 0 & 6 & 27 & $33^{*}$ & 0 & 0 & 0 & $33^{*}$ & 0 & 0 & 0 \\
\hline & & & & & \multicolumn{4}{|c|}{$\chi 2=60.000$} & \multicolumn{4}{|c|}{$\chi 2=72.660$} \\
\hline & & \multicolumn{3}{|c|}{$P=0.116$} & \multicolumn{4}{|c|}{$P<0.001$} & \multicolumn{4}{|c|}{$P<0.001$} \\
\hline
\end{tabular}

Note: *Compared to CSEA group, the cases of nausea and the modified Bromage scores of LR group were significantly lower on the end of anesthesia and on the end of operation $(P<0.05)$. 
Table 4: Adverse reaction and different recording time in two group.

\begin{tabular}{|c|c|c|c|c|c|c|}
\hline & N & Nausea & No-nausea & $\begin{array}{c}\text { Duration of } \\
\text { anesthesia (min) }\end{array}$ & $\begin{array}{c}\text { Onset of } \\
\text { operation(min) }\end{array}$ & $\begin{array}{c}\text { Duration of } \\
\text { Operation(min) }\end{array}$ \\
\hline CSEA & 27 & 13 & 14 & $16.04 \pm 1.51$ & $25.04 \pm 1.4$ & $55.70 \pm 4.78$ \\
\hline LR & 33 & $4^{*}$ & $29 *$ & $13.21 \pm 1.52^{*}$ & $23.24 \pm 1.60^{*}$ & $55.18 \pm 4.40$ \\
\hline & & \multicolumn{2}{|c|}{$\chi 2=9.492$} & $\mathrm{t}=7.203$ & $\mathrm{t}=4.567$ & $\mathrm{t}=0.439$ \\
\hline & & \multicolumn{2}{|c|}{$P=0.002$} & $P<0.001$ & $P<0.001$ & $P=0.662$ \\
\hline
\end{tabular}

Note: *Compared to CSEA group, the duration of local anesthesia and onset of operation in LR group were significantly shorter $(\mathrm{P}<0.05)$.

Table 5: Apgar score of neonates in two groups.

\begin{tabular}{|c|c|c|c|}
\hline & N & 1min & 5min \\
\hline CSEA & 27 & $8.56 \pm 0.58$ & $9.63 \pm 0.49$ \\
\hline LR & 33 & $8.73 \pm 0.52$ & $9.58 \pm 0.50$ \\
\hline$t$ & & -1.215 & 0.417 \\
\hline$P$ & & 0.229 & 0.678 \\
\hline
\end{tabular}

\section{Discussion}

CSEA and EA are commonly performed in patients undergoing ECS, with benefits and risks attributable to each [15]. Although CSEA was much more popular in CS due to its rapid onset of anesthesia, both of them were similar with regard to neonates Apgar scores and maternal satisfaction [16]. Based on these outcomes, our study was consistence with the previous. This study demonstrated that EA and CSEA were similar on anesthesia effect and neonates' Apgar score. More focuses are on rapid onset and more reliability of CSEA, however EA was primarily when minimizing patients' risk of hypotention. The effect of EA or CSEA on maternal blood pressure is controversial, but SA is believed more likely with hypotention, therefore more studies might be needed to compare hemodynamics changes between EA and CSEA [17]. In 2004 J Parsons reviewed SA and EA, the conclusion demonstrated SA was more likely to induce hypotension [18], moreover recently SA was still believed to be associated with hypotension [19]. In the present study, compared to EA, CSEA induced lower SBP, but it returned to similar level in two groups after $30 \mathrm{~min}$ of anesthesia. The hypotension relating SA was also verified by clinical trail [20]. Meanwhile, because hypotension is the major causative factor for intraoperative nausea and vomiting [21], this could explain reason of more nausea in CSEA group in this study.

The present study also paid more attention on differences of duration of anesthesia maneuver, onset of operation and duration of operation between two anesthesia methods, which differed from the previous studies that focused on the onset of neuraxial anesthesia.

This study showed that EA spent about 2min less than CSEA on maneuver duration and onset of operation. This might be that hyperbaric ropivacaine must be compounded on the spot.
EA might take 19min to T4 level [22], so in ECS rapid onset of anesthesia might be nonsense. Actually in some region, EA was still performed in urgent CS [23]. Additionally, lidocaine with rapid onset of anesthesia might shorten the delayed onset of EA [24]. The spinal dural is a normal anatomic structure to protect subdural matter, it is believed dural puncture may increase the possibility of injury and subdural infection. This study also demonstrated EA associated with delayed onset and shorter duration of motor block. This effect might help patients mobilize earlier and prevent complications related to delayed motor block such as urinary retention, and patient's discomfort with immobilization [25]. Thus higher maternal satisfaction might be with EA than SA for ECS [26]. Intrathecal injection of $2 \mathrm{ml}$ of $0.75 \%$ plain ropivacaine could produce longer than $100 \mathrm{~min}$ complete motor block [27], therefore modified Bromage score was much higher after about $80 \mathrm{~min}$ of anesthesia maneuver and operation with CSEA. However EA with lidocaine produced much shorter motor blockade, this was similar to the results of Seow's study [28].

\section{Conclusion}

Because of similar anesthesia effect, both of EA and CSEA were suitable for ECS. However, EA with lidocaine and low dose of ropivacaine could produce more stable hemodynamic, less nausea and shorter motor blockade, without delayed onset of operation. So it was more effective and simple anesthesia method for ECS. EA is the base for CSEA, therefore ongoing training EA is integral to the safe provision of the latter.

\section{Limitation}

There were some limitations in the present study. Firstly, because sample size was small, thus the results should be cautiously evaluated and large sample size study should be needed in future. 
Moreover the detailed technique of anesthesia in ECS should be selected according to the experience of the anesthetist, patient's clinical presentation, as well patient's wishes. Secondly, because supplement of analgesia agent were infrequently administered, thus it was not compared in two groups of this study. If the sample size was increased, the supplement of analgesia medicine should be compared to distinguish anesthesia effect between two anesthesia techniques.

\section{Conflict of Interest}

All authors declare that they have no conflict of interests.

\section{Author Contributions}

$\mathrm{XJ}$ designed the research and analyzed the data. WHJ performed anesthesia for all patients and wrote the manuscript. BY monitored and evaluated all parturient patients.

\section{Ethics Approval and Consent to Participate}

Our study was approved by the Ethic Committee of Capital Medical University Beijing Tongren Hospital. And the written informed consent was obtained from all included patients.

\section{Acknowledgment}

The authors would thank all staffs in the department of anesthesiology and operating-room of Capital Medical University Beijing Tongren Hospital.

\section{References}

1. Murouchi T, Iwasaki S, Yamakage M (2016) Quadratus lumborum block: analgesic effects and chronological ropivacaine concentrations after laparoscopic surgery. Reg Anesth Pain Med 41(2): 146-150.

2. Liu Q Wang JJ, Yan HY, Wang YQ Qin CW, et al. (2017) Safety and pharmacodynamic mechanism of ropivacaine lumbar anesthesia in Cesarean section. J Biol Regul Homeost Agents 31(1): 71-76.

3. Olapour A, Akhondzadeh R, Rashidi M, Gousheh M, Homayoon R (2020) Comparing the Effect of Bupivacaine and Ropivacaine in Cesarean Delivery with Spinal Anesthesia. Anesth Pain Med 10(1): e94155.

4. Zhao N, Xu J, Li XG, Walline JH, Li YC, et al. (2020) Hemodynamic characteristics in preeclampsia women during cesarean delivery after spinal anesthesia with ropivacaine. World J Clin Cases 8(8): 1444-1453.

5. Zhang W, Wu H (2017) ED50 of intrathecal ropivacaine for cesarean section under prophylactic infusion of phenylephrine: A consort study. Medicine (Baltimore) 96(44): e8319.

6. Ngan Kee WD (2010) Prevention of maternal hypotension after regional anaesthesia for caesarean section. Curr Opin Anaesthesiol 23(3): 304 309.

7. Wang HZ, Chen HW, Fan YT, Jing YL, Song XR, et al. (2018) Relationship Between Body Mass Index and Spread of Spinal Anesthsia in Pregnant Women: A Randomized Controlled Trial. Med Sci Monit 24: 6144-6150.

8. Van de Velde M (2019) Low-dose spinal anesthesia for cesarean section to prevent spinal-induced hypotension. Curr Opin Anaesthesiol 32(3): 268-270.

9. Lv M, Zhang P, Wang Z (2018) ED(50) of intrathecal ropivacaine for cesarean delivery with and without epidural volume extension with normal saline: a randomized controlled study. J Pain Res 11: 2791-2796.

10. Xu W, Xiao F, Zhang Y, Liu L, Chang X (2018) ED50 and ED95 of intrathecal hyperbaric ropivacaine for parturients undergoing cesarean section with prophylactic infusion of phenylephrine: A Prospective dose-finding Study. Medicine (Baltimore) 97(50): e13727.

11. Riley ET, Cohen SE, Macario A, Desai JB, Ratner EF (1995) Spinal versus epidural anesthesia for cesarean section: a comparison of time efficiency, costs, charges, and complications. Anesth Analg 80(4): 709-712.

12. Al-Husban N, Elmuhtaseb MS, Al-Husban H, Nabhan M, Abuhalaweh H, et al. (2021) Anesthesia for Cesarean Section: Retrospective Comparative Study. Int J Womens Health 13: 141-152.

13. Felfernig M, Marhofer P, Weintraud M, Huber G, Duma A, et al. (2010) Use of ropivacaine and lidocaine for axillary plexus blockade. Afr J Paediatr Surg 7(2): 101-104.

14. Bi YH, Wu JM, Zhang YZ, Zhang RQ (2020) Effect of different doses of intrathecal dexmedetomidine as an adjuvant combined with hyperbaric ropivacaine in patient undergoing cesarean section. Front Pharmacol 11: 342.

15. Liu J, Wang Y, Ma W (2018) Shivering prevention and treatment during cesarean delivery under neuraxial anesthesia: a systematic review. Minerva Anestesiol 84(12): 1393-1405.

16. Huang CH, Hsieh YJ, Wei KH, Sun WZ, Tsao SL (2015) A comparison of spinal and epidural anesthesia for cesarean section following epidural labor analgesia: A retrospective cohort study. Acta Anaesthesiol Taiwan 53(1): 7-11.

17. Hood DD, Curry R (1999) Spinal versus epidural anesthesia for cesarean section in severely preeclamptic patients: a retrospective survey. Anesthesiology 90(5): 1276-1282.

18. Ng K, Parsons J, Cyna AM, Middleton P (2004) Spinal versus epidural anaesthesia for caesarean section. Cochrane Database Syst Rev 2: CD003765.

19. Šklebar I, Bujas T, Habek D (2019) Spinal anaesthesia-induced hypotension in obstetrics: prevention and therapy. Acta Clin Croat 58(1): 90-95.

20. Huang B, Huang Q, Hai C, Zheng Z, Li Y, et al. (2019) Height-based dosing algorithm of bupivacaine in spinal anaesthesia for decreasing maternal hypotension in caesarean section without prophylactic fluid preloading and vasopressors: study protocol for a randomised controlled noninferiority trial. BMJ Open 9(5): e024912.

21. Shin DW, Kim Y, Hong B, Yoon SH, Lim CS, et al. (2019) Effect of fentanyl on nausea and vomiting in cesarean section under spinal anesthesia: a randomized controlled study. J Int Med Res 47(10): 4798-4807.

22. Choi DH, Kim JA, Chung IS (2000) Comparison of combined spinal epidural anesthesia and epidural anesthesia for cesarean section. Acta Anaesthesiol Scand 44(2): 214-219.

23. Sarı MA, Küçükgüçlü S, Özbilgin Ş, Günenç FS, Mercan S, et al. (2015) Retrospective Evaluation of Anaesthetic Techniques for Caesarean. Turk J Anaesthesiol Reanim 43(6): 373-380.

24. Powell MF, Jarzombek KW, Venhuizen KJ, Tubinis MD, Morgan CJ, et al. (2019) Comparing Bupivacaine, Lidocaine, and a Combination of Bupivacaine and Lidocaine for Labor Epidural Activation: A Prospective, Randomized, Double-Blind Study. Asian J Anesthesiol 57(2): 55-60.

25. Yun MJ, Kwon MY, Kim DH, Lee JW (2014) Combined spinal-epidural anesthesia using a reduced-dose of spinal bupivacaine and epidural top up leads to faster motor recovery after lower extremity surgeries. Korean J Anesthesiol 66(1): 28-33.

26. Morgan PJ, Halpern S, Lam-McCulloch J (2000) Comparison of maternal satisfaction between epidural and spinal anesthesia for elective Cesarean section. Can J Anaesth 47(10): 956-961.

27. Kumar SS, Talwar V, Gupta P, Gogia AR (2018) Comparison of the Efficacy of Intrathecal Isobaric Ropivacaine and Bupivacaine in Day Care Knee Arthroscopy: A Randomized Controlled Trial. Anesth Essays Res 12(4): 859-864.

28. Seow LT, Lips FJ, Cousins MJ, Mather LE (1982) Lidocaine and bupivacaine mixtures for epidural blockade. Anesthesiology 56(3): 177-183. 
ISSN: 2574-1241

DOI: 10.26717/BJSTR.2021.36.005866

Jun Xiong. Biomed J Sci \& Tech Res

(c) (P) This work is licensed under Creative

Submission Link: https://biomedres.us/submit-manuscript.php

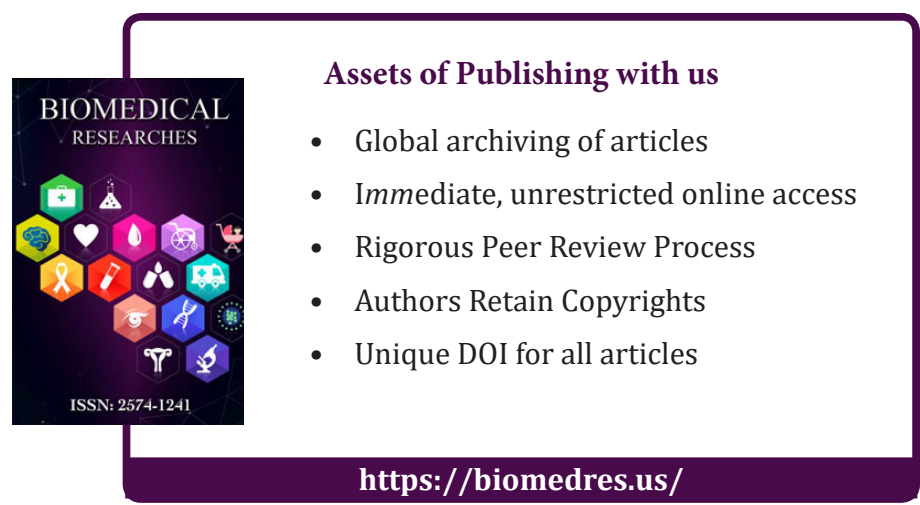

